\title{
APPLICATION OF MICROTEXTURE DETERMINATION USING EBSD TO NON CUBIC CRYSTALS
}

\section{J DINGLEY, A DAY and A BEWICK}

H H Wills Physics Laboratory, University of Bristol, Tyndall Ave. Bristol BS81TL UR

\section{INTRODUCTION}

The technique for obtaining individual crystal orientation using electron backscatter diffraction (EBSD) in the scanning electron mucroscope, first demonstrated by Venables and Harland in $1973^{1}$ and adpted for on-line texture determination by Dingley et al. in $1984^{2}$, is well established for cubic materials, Randle et al. ${ }^{3}$. Hjelen and Nes 4 Juul Jensen and Randle ${ }^{5}$. Extension of the technjque to hexagonal and rhombohedral crystals was reported briefly by Dingley in 19876,7 . This paper presents a full account of the general procedure for obtaining on-line orientation measurements from non cubic crystals with examples from hexagonal zircaloy and orthorhombic BiSrCuCaO high temperature superconducting materjal.

It has also been recognised for some time that pole figure representation of the orjentation data obtained using the EBSD method us not jdeal, and an alternative representation, a Frank Rodrigues (PR) figure, was presented at the 1987 ICOTOM meeting. Frank. Here is presented a further extension of this representation in which the absolute and relative orjetations of crystals are displayed in a micrograph of the grasn structure by a colour specific to one pount in the FR figure.

\section{EXPERIMENTAL PROCEDURE}

Details of the EBSD technique have been pubisshed previously Dingley et al. 9 Briefly, the sample us mounted an an SEM incluned so that its surface normal pounts 70 from the uncident beam and towards a vertucal phosphor screen, $50 \mathrm{~mm}$ in duameter and piaced 50 min fron the specimen. To obtajn a diffractuon pattern the electron beam of the SEM is positjoned as a stationary probe within the grain of unterest. Kikuch type diffraction occurs withm the specimen and the diffracted beams which exit from the top surtace of the specimen are imaged on the phosphor screen. The spatual resolution of the technoue is $200 \mathrm{~mm}$ and the angular precision in orientation measurement 1 to 2, depending on the accuracy of the calibration. The phosphor screen is viewed using a low light level television camera and on-lune orjentation measurement is made by superimposing onto the TV image of the pattern a computer generated cursor. The cursor is positioned successively on designated zone axes in the pattern and by measuring the locations of these zone axes the pattern can be indexed automatically and the orientation determined. The diffraction pattern centre 
and specimen to screen distance, necessary to interprete the patterns, are determined from prior measurements of zone axis positions on a calibration crystal mounted in the microscope at a known orientation. Advances in execution of the technique for cubic crystals have permitted meaurement of grientation without the need to input the indices of the selected zone axes, Dingley 10 and in a more advanced technique still the pattern is jdentified using computer based pattern recognition procedures, Juul Jensen and Schmidt 11 .

\section{NON-CUBIC CRYSTALS}

Diffraction patterns from cubic crystals are relatively easy to recognise because the hugh symmetry means that each zone axis type is repeated often so that only a few different types need to be remembered by the operator or recognised by the computer. Each is distinguished by the arrangement of Kikuchi lines passing through the zone axis. As the crystal symmetry decreases more zone axes types need to be recognised and whereas this is still relatively simple for hexagonal crystals, distinguishing between [100] and $[001]$ can become very difficult in orthorhombic crystals for example, especially when the a:b ratio is close to 1. Fig.1 shows a pattern from hexagonal silicon carbide. Indexing is clearly difficult but with the use of a set of reference photographs for a specific crystal structure on-line operator recognition becomes $100 \%$ successful. Fig. 2 shows a diffraction pattern from the high temperature BistCuCaO superconducting material taken close to [001]. The material was prepared by cladding in silver followed by severe cold reduction. Subsequent high temperature annealing un oxygen both recrystallises the material and renders it superconducting. Whereas it was s]mple to distinguish the [001] axis it was not possible to distinguish between $[100]$ and $[010]$ due to the $a: b$ ratio being 0.01 .

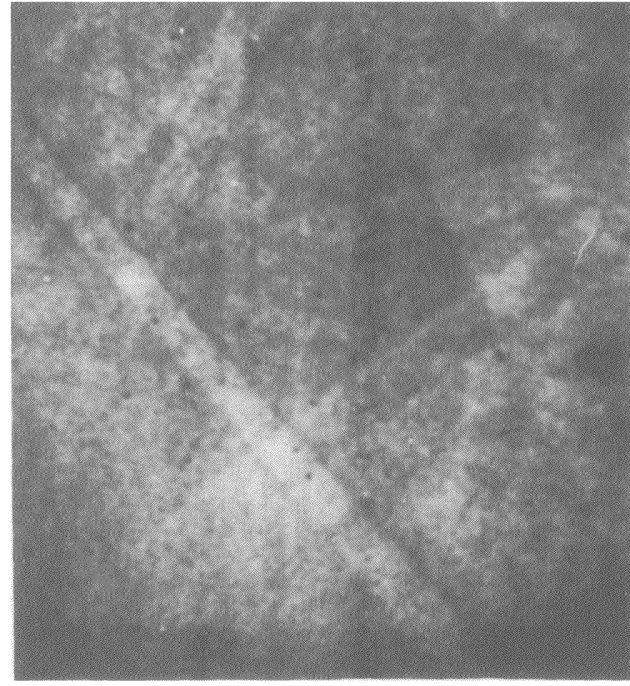

Fig.l EBSP from hexagonal zircaloy

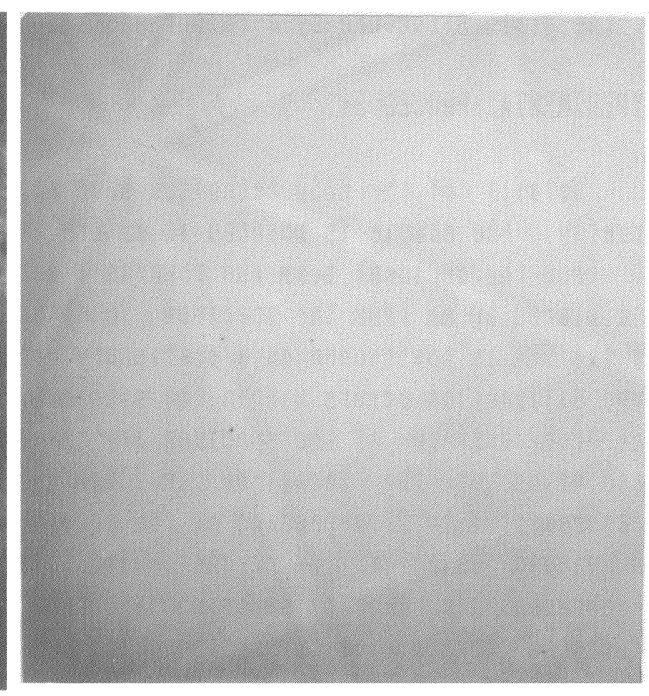

Fig2. EBSP from orthorhombic BisrCaCuO 
RESULTS

On-line orientation measurement of the hexagonal crystals presented no difficulties. Specific zone axes were recognised by the operator. Miller indices of the selected axes were input to the computer and the locations of the axes determined by positioning the computer generated cursor on them. An example of data obtained from annealed zircaloy is presented in the pole figure, fig. $3 a, b$. The basal plane [0001] and pyramidal plane [1010] orientations are shown. The basal plane X-ray determined pole figures for the same specimen are shown in fig3c.d. for comparison. Considering that only 230 grain orientation were measured to determine the BBSD pole figure the agreement between EBSD and X-ray data is extremely good.
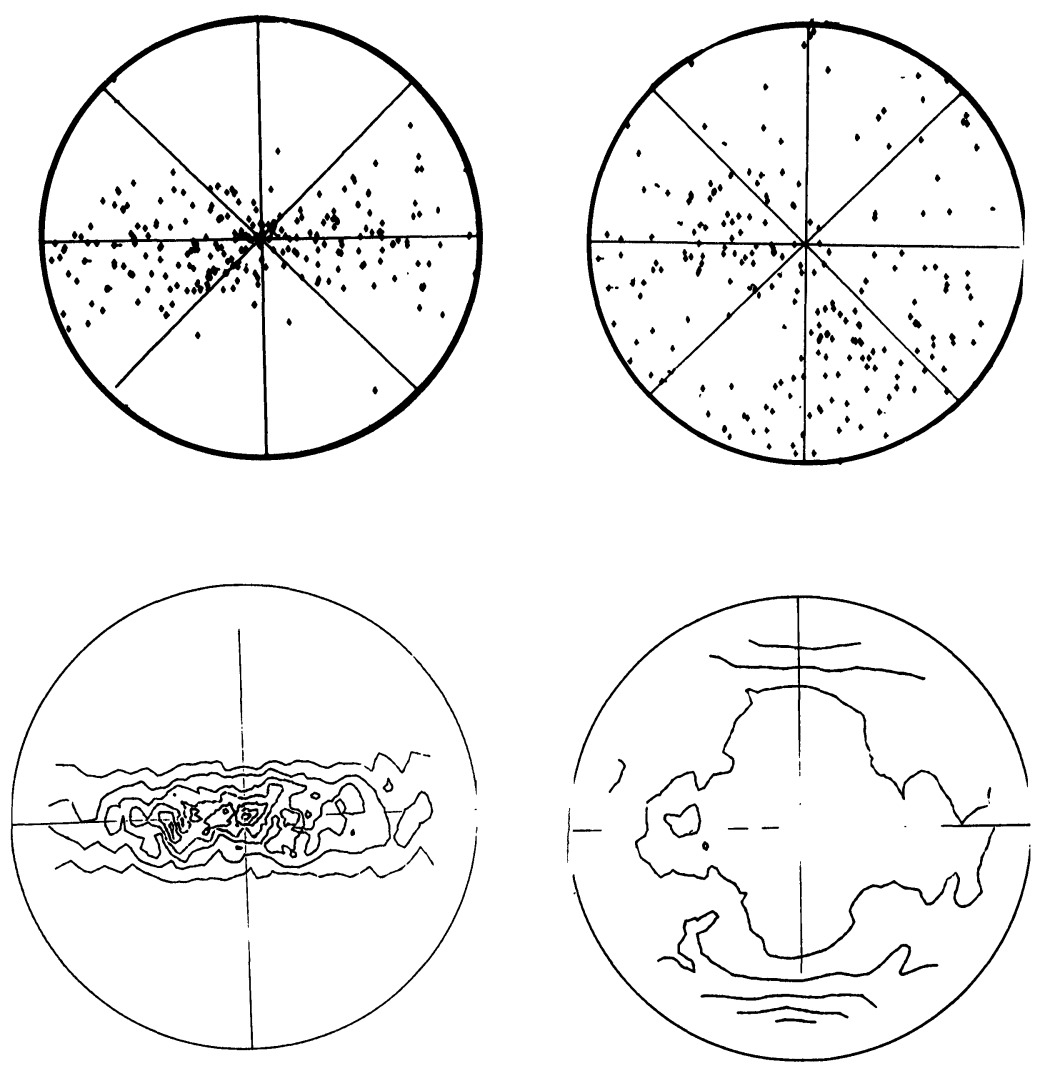

Fig. 3,a,b. [0001] and [1010] pole figures for hexagonal zircaloy obtaned using EBSF Fig. $3 . c, d$. Corresponding tigures to $3 a$ and $3 b$ obtained using $x$-ray diffraction

On-lune orientation measurement of Bisrcacuo was more difticult but successful because as the material was highly textured all grauns examined presented the r [001] axis towards the screen. It was also possible to distinguush on-lune between the broad (110) and narrow [010) Kikuchi bands passung through (001). By adapting the program to accept bana posutions rather than zome axes, orsentatuon determination could thus be achieved. The results are presented in the pole figure, fig. $4 a$. where the $[001\}$ poles only are plotted. The highly textured nature of the material is 
shown. Because it was not possuble to distinguish between $\{100\}$ and $[010\}$ the data for these two axes are combined in fig. $4 b$.
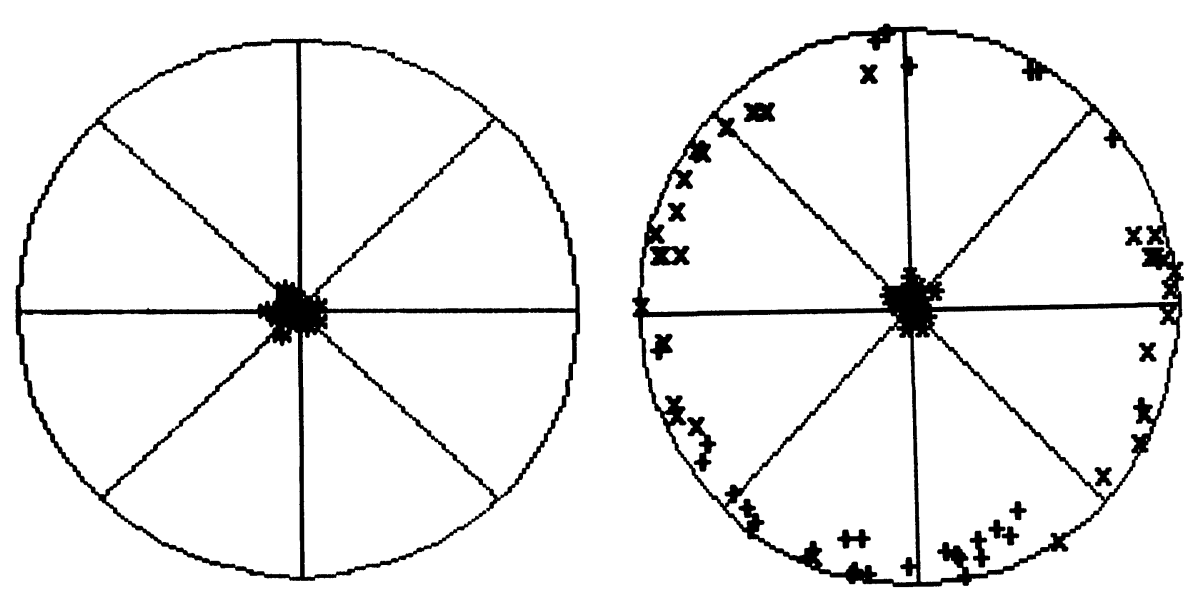

Pig. 4. Poles figures obtajned using EBSP for BisrCaCuo, a. 1001$\}$ poles only. b. $[001],[100]$ and $[010]$ poles.

FRANK RODRIGUES FIGURES

To determine the absolute orientations of crystals by the combined inspection of figs $3 a$ and $3 b$ or $4 a$ and $4 b$ it is clearly an mpossible task. However, the very nature of the EBSD technique provides the absolute orientation of each crystal examined and it is only nessary to produce a means of representing these measurements in pictorial form. A means was described by Frank which we now refer to as Frank Rodrigues figures. For each crystal the rotation axis and rotation angle are calculated that would rotate the crystal so that its reference axes were made parallel to the reference axes of the specimen. These parameters are given by the Eigen vector and the trace of the orientation matrix respectively defining each crystal. The magnitude of the rotation axis is made equal to the modulus of the vector multiplied by $\tan ($ halt the rotation angle). Each vector is plotted in a figure whose origin is the centre of the figure and whose axes are parallel to the specimen reference axes. Such a plot is shown for grain orientations obtained from recrystallised pure nickel in fig.5a. The figure has the advantage that it exhibits the symmetry of the crystal structure and is bounded by a surface also related to the crystal symetry, Corresponding figures for the zircaloy aterial is shown in fig. $5 b$

\section{ORIENTATION IHAGING}

The combination of scanning electron nicroscopy and EBSD affords a unique opportunity to represent the spatial distribution of crystal orjentations by superimposing on a micrograph of the specimen the individual crystal orientations. 

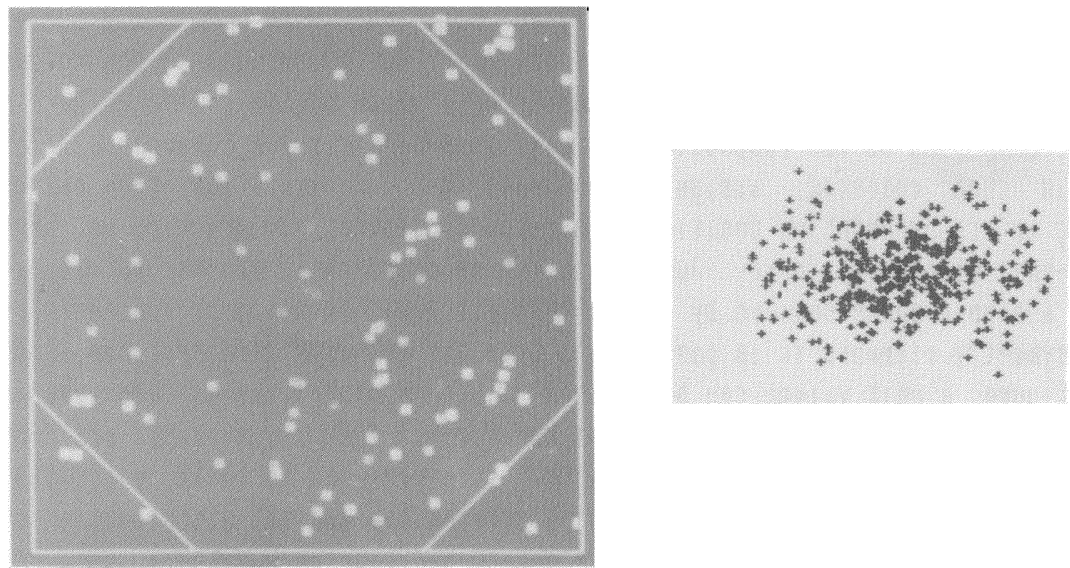

Fig. 5a Rodrigues Frank map for Ni grains. Figs.b Corresponding map for zurcaloy.
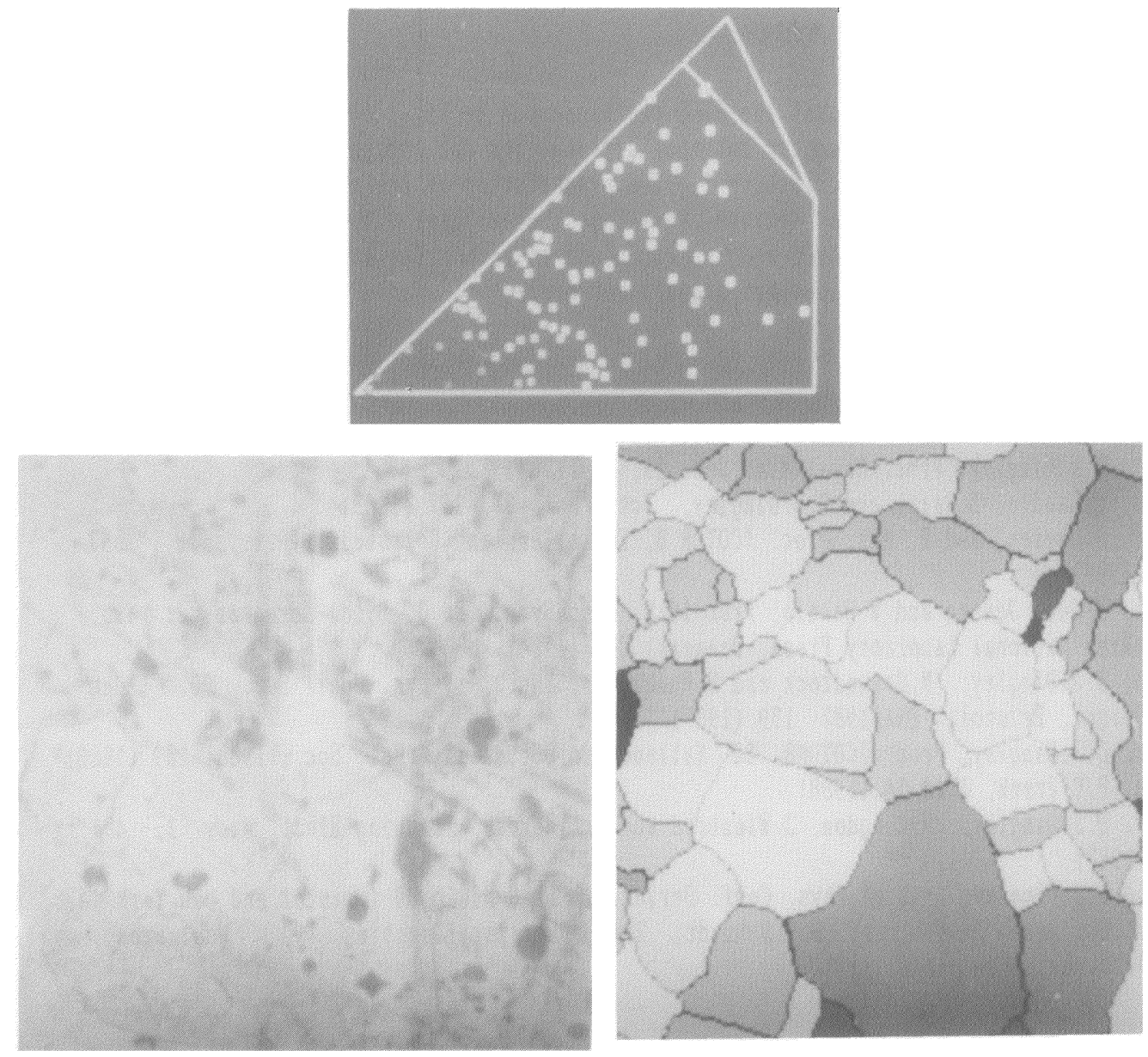

Pig. 6a $\mathrm{Hi}$ grain orientations represented in unit volume of $\mathrm{PR}$ figure.

Pig.6b (upper), micrograph of $\mathrm{Ni}$ grains, (lower) orientation image of same grains. 
As before, the individual orientations are defined in the FR figure. Each $x, y, z, a X] S$ of the figure is assigned the colour red, biue or green, the brightness of the colour increasing with distance from the origin. A point withun the figure will then have a unique colour depending on the respective contributions of $j$ ts $x, y, z$ components. The aicrograph is then coloured, assigning to each grain orientation the colour corresponding to its orientation position withon the FR figure. In practice we have used a sub-unit of the FR figure. Just as in the stereographich projection it is possible to assign a space bounded by [001], [110], (111) within which by because of the crystal symmetry elements it is possible to transter all crystal durections, so. in the FR figure, a unit volume can be defined. In this case it is an odd shaped fugure, see fig.6. constitutung 1/48th. of the total. The $x, y, z$ axes are not orthogonal and the sides are not of equal length. $x$ is parallel to $[001]$, y to $[1101$ and $z$ to [111]. Fig. 6 also includes a micrograph showing the spatial distrubution of the grains from which the orientations were determined together with a coloured superposition of the micrograph and the orientations. It is seen that in this case not only is the texture randon but so is the spatjal distribution of orjentations, i.e there is no clustering of grains with particular orientations

CONCLUSIONS

It has been shown that the EBSD technique can be extended to include crystals with lower than cubic symetry but that diffjculties and ambiguities can arsse where $a: b$ or $b: c$ ratios are close to 1. The presentation of the orientations in Frank Rodriges map form has obvious advantages over pole fugure representation, particularly for non cubjes and its extension to permit the imaging of the spatial distribution of orientations has significant merit.

\section{REFERENCES}

1. J.A. Venables and C.J.Harland, Phil. Mag. 27, 1193 (1973)

2. D.J. Dingley, Proc. Royal Mic. Soc. 19, 74 (1984)

3.V. Randle, B. Ralph and D.J.Dingley, Acta. Met, 36, 267 (1988)

4. J.Hjelen and E. Nes, Proc. ICOTOK 8, Ed Kallend and Gottstein, (Met. Soc.) 597 (1988)

5 D. Juul Jensen and V Randle, Proc 10th. Riso Symp., Ed J. Bilde-Sorensen et al., (Riso National Laboraory Press, Denmark) 325 (1984)

6. D.J.Dingley, N.Gravestock and H. Rothstein, Inst. of Phys. Conf. Ser. 90. (Adam Hjlger, Bristol) BMaG1987 139 (1987)

7. D.J.Dingley, Proc. ICOTOH8, Ed. Rallend and Gottstein, (Met. Soc., 1988) 189 (1988)

8. F.C. Frank, Ibid 54 (1988)

9. D.J.Dingley, M.Longdon, J.Wienbren and J.Alderman, Scanning Elect. Mic. 1, 451 (1987)

10 D.J. Dingley, Inst. of Phys. Conf. Series. 98 BMAG-HICRO 89 (Bristol and New York) 473, (1989) 11 D. Juul Jensen and N.H.Schmidt, Porc. Recrystallisation 190, Noolongong, Ausatralia, In press, (1990) 